\title{
Distribution and Determinants of Endoscopic third ventriculostomy (ETV) at a Specialized Hospital in Dhaka City
}

\author{
SK Sader Hossain ${ }^{1}$, Md Abdullah Alamgir², Ferdous Ara Islam³, Sheikh Mohammed Ekramullah ${ }^{4}$, \\ Shudipto Kumar Mukharjee ${ }^{5}$, ATM Asadullah ${ }^{6}$, Sarwar Morshed Alam 7 , Kalimuddin ${ }^{8}$, \\ Kaiser Haroon ${ }^{9}$, Misbah Uddin Ahmed ${ }^{10}$, Mahmudul Huq ${ }^{11}$
}

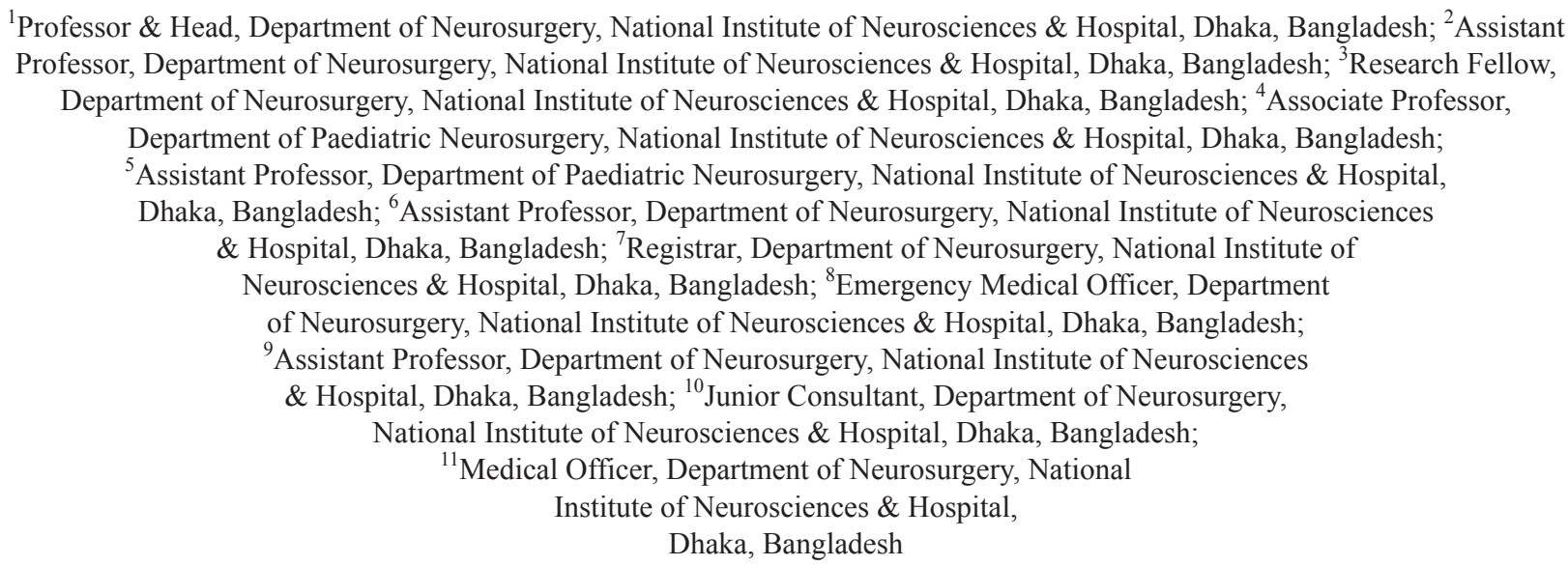

Received on: 24 July 2014; Reviewed on: 26 September 2014; Published on: 1 January 2015

\begin{abstract}
Background: Endoscopic third ventriculostomy (ETV) is the process of intra cranial CSF diversion to relieve the pressure inside the ventricles. This allows the cerebrospinal fluid to flow directly to the basal cisterns, thereby shortcutting any obstruction. It is used as an alternative to a cerebral shunt surgery. Objectives: To observe the Endoscopic third ventriculostomy (ETV) with causal factors and outcome. Methodology: The study was conducted in the Department of Neurosurgery in National Institute of Neurosciences (NINS) during the period from June, 2013 to August, 2014. All the study subjects included in the study were selected for endoscopic third ventriculostomy (ETV) following clinical and radiological diagnosis of hydrocephalus or raised ICP irrespective of age, sex and causal factors. The patients were followed post operatively to follow the outcome. Result: ETV was performed among 38 males and 35 females with a mean age of 24.67 years. Three major causal factors for ETV were aqueductal stenosis, posterior fossa SOL and CP angle tumour observed in $25(34.3 \%), 22(30.2 \%), 11(15.1 \%)$ cases respectively. The successful ETV was done in $49(67.1 \%)$ patients varied widely by diagnosis and patient age. Other $32.9 \%$ had suffered from several complications like local CSF drainage, local infection, meningitis and subarachnoid haemorrhage and treated conservatively. Conclusion: Endoscopic third ventriculostomy (ETV) is a safe and successful procedure in the management obstructive hydrocephalus. [J Natl Inst Neurosci Bangladesh 2015;1(1): 5-7]
\end{abstract}

Keywords: endoscopic third ventriculostomy (ETV), obstructive hydrocephalus, aqueductal stenosis.

Corresponding author: Dr. Ferdous Ara Islam, Research Fellow, Department of Neurosurgery, National Institute of Neurosciences \& Hospital, Dhaka, Bangladesh; Email: islamaraferdous@yahoo.com; Cell No.: +8801777403057

Conflict of interest: Authors declared no conflict of interest

Funding of the study: None

Contribution of the authors: SKSH, MAA \& FAI have given the concept, manuscript editing, revising as well as intellectual thoughts. Rest of the authors as well as the other mentioned authors have contributed during surgery.

How to cite this article: Hossain SKS, Alamgir MA, Islam FA, Ekramullah SM, Mukharjee SK, Asadullah ATM, Alam SM, Kalimuddin, Haroon K, Ahmed MU, Huq M. Distribution and Determinants of Endoscopic third ventriculostomy (ETV) at a Specialized Hospital in Dhaka City, J Natl Inst Neurosci Bangladesh 2015;1(1): 5-7

\section{Introduction}

Endoscopic neurosurgery was first introduced in Bangladesh at 2005. Following the establishment of National Institute of Neurosciences (NINS) in Dhaka, Bangladesh the endoscopic Neurosurgery begins a new era. The procedure was first started here at June, 2013. Endoscopic third ventriculostomy (ETV) is one of the major procedure had been done to reduce mortality or morbidity of the raised intracranial pressure (ICP) patients.

Endoscopic third ventriculostomy (ETV) is the process of intracranial CSF diversion to relieve the pressure inside the 
ventricles. In this process a fenestration is made in the thinned floor of third ventricle which is an attempt to bypass the obstruction in the aqueduct of the Sylvius ${ }^{1}$. The process is not a cure for hydrocephalus rather a process for an alternative to shunt surgery ${ }^{1,2}$. The first open third ventriculostomy was done in 1922. One year later, mixter carried out first endoscopic procedure in neurosurgery by using a small ureteroscope $e^{3,4}$. Now a days rigid and fiber optic endoscopes both are used for ETV. In this Institute, rigid endoscopes are used for endoscopic procedures till date. The aim of this study was to observe the endoscopic third ventriculostomy (ETV) performed at NINS with causal factors and outcome of the patients.

\section{Methodology}

This was a retrospective study. The study was conducted in the Department of Neurosurgery at National Institute of Neurosciences (NINS) during the period from June, 2013 to August, 2014. All the study subjects included in the study were selected for endoscopic third ventriculostomy (ETV) following clinical and radiological diagnosis of hydrocephalus or raised ICP irrespective of age, sex and causal factors.

\section{Results}

The majority of cases from 1-10 years comprised 17 $(23.3 \%)$ cases, followed by $16(21.9 \%)$ cases from $11-20$ years and 14 cases (19.2\%) from 21-30 years (Table 1).

Table 1: Age distribution of the patients $(n=73)$

\begin{tabular}{lcc}
\hline Age Group & Frequency & Percentage (\%) \\
\hline Below 1 year & 4 & 5.5 \\
1-10 years & 17 & 23.3 \\
11-20 years & 16 & 21.9 \\
21-30 years & 14 & 19.2 \\
31-40 years & 7 & 9.6 \\
41-50 years & 5 & 6.8 \\
51-60 years & 9 & 12.3 \\
61-70 years & 1 & 1.4 \\
Total & $\mathbf{7 3}$ & $\mathbf{1 0 0 . 0}$ \\
\hline
\end{tabular}

Among 73 study subjects, 38 (52.05\%) were male and 35 (47.95\%) were female (Table 2$)$. The main causal factors for ETV were aqueductal stenosis $(34.3 \%)$ and posterior fossa SOL (30.2\%) (Table 3).

Table 2: Sex distribution of the patient $(n=73)$

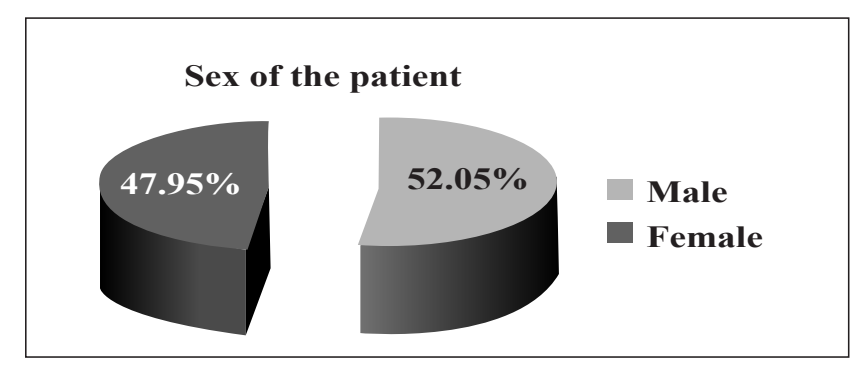

Among 73 ETV cases 49 (67.1\%) were functioning successfully. Other 24 cases had complications included local CSF leakage in $12(16.4 \%)$ cases, local infections were present in $10(13.7 \%)$ cases. One case developed meningitis and other one developed sub arachnoid Haemorrhage postoperatively (Table 4).

Table 3: Distribution of causal factors of raised ICP $(n=73)$

\begin{tabular}{lcc}
\hline Causal factors & Frequency & Percentage \\
\hline Aqueductal stenosis & 25 & 34.3 \\
Posterior fossa SOL & 22 & 30.2 \\
CP angle Tumour & 11 & 15.1 \\
Cerebellar SOL & 3 & 4.1 \\
Ventricular Tumour & 3 & 4.1 \\
Shunt failure & 4 & 5.4 \\
Arachnoid cyst & 4 & 5.4 \\
Colloid cyst & 1 & 1.4 \\
Total & $\mathbf{7 3}$ & $\mathbf{1 0 0 . 0}$ \\
\hline
\end{tabular}

\section{Discussion}

In this study male female ratio was 1.08. Majority of the patients were from first three decades of life. The highest number of patients were 17 (23.3\%) from 1-10 years of age. There were $4(5.5 \%)$ patients below 1 year of age. ETV is common in children in treatment of hydrocephalus ${ }^{1}$.

Table 4: Outcome following ETV $(n=73)$

\begin{tabular}{lcc}
\hline Outcome & Frequency & Percentage \\
\hline Functioning ETV & 49 & 67.1 \\
Complications & & \\
Local CSF leakage & 12 & 16.4 \\
Local infection & 10 & 13.7 \\
Meningitis & 1 & 1.4 \\
Sub arachnoid Haemorrhage & 1 & 1.4 \\
Total & $\mathbf{7 3}$ & $\mathbf{1 0 0 . 0}$ \\
\hline
\end{tabular}

The wide range of acceptance of ETV in treatment of Hydrocephalus $^{5}$. ETV is a short duration, noninvasive procedure. Obstructive hydrocephalus is the main indication for endoscopic third ventriculostomy, but this procedure is also currently used to treat a wide range of hydrocephalic patients. It is generally considered that aqueductal stenosis is a precursor to non-communicating hydrocephalus, as the blockage of the aqueduct would result in the accumulation of CSF seen in hydrocephalus. Aqueductal stenosis can be developed by tumour compression also $^{6}$. Analysis of the preliminary results from this retrospective study conducted in a significant number of patients' shows that endoscopic third ventriculostomy is a feasible and safe method of treating hydrocephalus associated with posterior fossa tumors. When performed before posterior fossa surgery, it had both a curative effect on intracranial hypertension and a prophylactic effect by preventing the development of hydrocephalus after tumor removal ${ }^{7}$.

According to several studies several complications may arise specific to ETV including bradycardia and asystole, visual obstruction, damage of ventricles, vascular sytems and fornices which were not observed in this study ${ }^{8}$. Current study revealed no such complications rather several general 
complications regarding ETV were observed included infection, meningitis, sub arachnoid haemorrhage, CSF leakage. The patients were treated conservatively and recovered well.

Previously ventriculo peritoneal shunting was done to reduce raised intra cranial pressure. Hydrocephalus was treated through VP shunt. But the complications of the shunt ranging from infection, mechanical shunt blockage, shunt malfunction, over drainage or under drainage of CSF fluid, shunt extrusion through the skin even through rectum ${ }^{9-10}$. Success rate of ETV depends upon Surgeon's good knowledge of third ventricle anatomy and experience with endoscopic surgery. High Success Rate $(>75 \%)$ observed post operatively in aqueductal stenosis, tumoural mass cases ${ }^{11}$. Current study revealed overall success rate was $67.1 \%$.

\section{Conclusion}

Though the study has been conducted in 1 year period and success rate was $67.1 \%$ post operatively. Further follow up required for full assessment of functioning Endoscopic third ventriculostomy (ETV).

\section{References}

1. Hydrocephalus Association.Fact sheet Endoscopic third ventriculostomy (ETV) Available at: http://www.hydroassoc.org/docs/FactSheets/FactsheetETV.pdf
2. Cinalli, G.; Spennato, P. Nastro, A. Aliberti, F. Trischitta, V. Ruggiero, C. Mirone, G. Cianciulli, E. Hydrocephalus in aqueductal stenosis. Childs Nervous System 2011;27(10): 1621-42

3. Cinalli G, Sainte-Rose C, Chumas P, Zerah M, Brunelle F, Lot G, Pierre-Kahn A, Renier D: Failure of third ventriculostomy in the treatment of aqueductal stenosis in children. J Neurosurg 1999;90;448-454,

4. Jones RFC, Stening WA, Brydon M, Paed M: Endoscopic third ventriculostomy. Neurosurgery 26;86-92, 1990

5. Oertel, Joachim M. K.; Vulcu, Sonja; Schroeder, Henry W. S.; Konerding, Moritz A.; Wagner, Wolfgang; Gaab, Michael R. Endoscopic transventricular third ventriculostomy through the lamina terminalis. Journal of Neurosurgery 2010;13(6): 1261

6. McMillan, J. J.; Williams, B. (June 1977).Aqueduct stenosis. Case review and discussion. Journal of Neurology, Neurosurgery, and Psychiatry 2012;40(6): 521-532 7. Roesetal CS. Management of hydrocephalus in pediatric patients with posterior fossa tumors: the role of endoscopic third ventriculostomy. JNeurosurg 2001;95:791-797

8. Charles Teo. Complicatios of endoscopic third ventriculostomy.Paediatric hydrocephalus (2005).Chapter 29.: 411-420. Available at: http://link.springer.com/chapter/10.1007\%2F978-88-470-2121-1_29

9. Sarguna P, Lakshmi V. Ventriculoperitoneal shunt infections. Indian Journal of Medical Microbiology.2006;24:52-54

10. Kulkarni a V, Drake JM, Lamberti-Pasculli M. Cerebrospinal fluid shunt infection: a prospective study of risk factors. Journal of Neurosurgery.2001;94:195-201

11. Ocuzc AT. Endoscopic Third Ventriculostomy: Indications, Surgical Technique and Potential Problems. Turkish Neurosurgery 2002;12: 65 - 73 